\title{
A rare example of low surface-brightness radio lobes in a gas-rich early-type galaxy: the story of NGC $3998^{\star}$
}

\author{
Bradley S. Frank ${ }^{1,2}$, Raffaella Morganti ${ }^{1,3}$, Tom Oosterloo ${ }^{1,3}$, Kristina Nyland $^{1,4}$, and Paolo Serra ${ }^{5}$ \\ ${ }^{1}$ Netherlands Institute for Radio Astronomy (ASTRON), Postbus 2, 7990 AA Dwingeloo, The Netherlands \\ 2 Department of Astronomy, University of Cape Town, Private Bag X3, 7701 Rondebosch, South Africa \\ e-mail: bradley@ast.uct.ac.za \\ 3 Kapteyn Astronomical Institute, University of Groningen, Postbus 800, 9700 AV Groningen, The Netherlands \\ ${ }^{4}$ National Radio Astronomy Observatory (NRAO), 520 Edgemont Rd., Charlottesville, VA 22903, USA \\ 5 CSIRO Astronomy and Space Science, Australia Telescope National Facility, PO Box 76, Epping, NSW 1710, Australia
}

Received 9 February 2016 / Accepted 5 May 2016

\begin{abstract}
We study the nearby lenticular galaxy NGC 3998. This galaxy is known to host a low-power radio AGN with a kpc-size one-sided jet and a large, nearly polar H I disc. It is therefore a good system to study to understand the relation between the availability of cold-gas and the triggering of AGNs in galaxies. Our new WSRT data reveal two faint, S-shaped radio lobes extending out to $\sim 10 \mathrm{kpc}$ from the galaxy centre. Remarkably, we find that the inner H I disc warps back towards the stellar mid-plane in a way that mirrors the warping of the radio lobes. We suggest that the polar H I disc was accreted through a minor merger, and that the torques causing it to warp in the inner regions are also responsible for feeding the AGN. The " $\mathrm{S}$ " shape of the radio lobes would then be due to the radio jets adapting to the changing angular momentum of the accreted gas. The extended radio jets are likely poorly collimated, which would explain their quick fading and, therefore, their rarity in galaxies similar to NGC 3998. The fuelling of the central super-massive black hole is likely occurring via "discrete events", suggested by the observed variability of the radio core and the extremely high core dominance, which we attribute to the formation and ejection of a new jet resulting from a recent fuelling event.
\end{abstract}

Key words. galaxies: active - galaxies: individual: NGC 3998 - ISM: jets and outflows - radio continuum: galaxies radio lines: galaxies - history and philosophy of astronomy

\section{Introduction}

Radio active galactic nuclei (AGN) are an important subpopulation of the general family of AGN which show a variety of different properties and morphologies. The low radio power group $\left(P_{1.4 \mathrm{GHz}}<10^{23} \mathrm{~W} \mathrm{~Hz}^{-1}\right.$; Sadler et al. 1989; Wrobel \& Heeschen 1991; Nyland et al. 2013) form the bulk of the radio emitting AGN population (Sadler et al. 2007; Best et al. 2005). Despite their low power, they can impact the ISM of the host galaxy as shown in a number of cases, e.g. NGC 1266 (Alatalo et al. 2011, 2015; Nyland et al. 2013, 2016), NGC 1433 (Combes et al. 2013) and IC 5063 (Morganti et al. 2015). It is therefore important that we study this population in more detail. Compared to powerful radio galaxies, low power radio sources tend to be more core dominated and have smaller spatial extents (Nagar et al. 2005; Baldi \& Capetti 2010; Baldi et al. 2015a; Nyland et al. 2016). Although still poorly understood, the brightness, morphology and scale of the radio emission may be related to a lower, or inefficient, supply of fresh gas or to weak/poorly collimated jets likely due to lower flow velocities and, therefore, are more subject to instabilities and entrainment (Nagar et al. 2005; Laing et al. 2011; Baldi et al. 2015a).

Considering the likely and widespread availability of gas, an important question is what the mechanisms are that can bring the gas to the inner regions and trigger the super-massive

\footnotetext{
* The reduced radiocontinuum image and HI datacube (FITS files) are only available at the CDS via anonymous ftp to

cdsarc.u-strasbg. fr (130.79.128.5) or via

http://cdsarc.u-strasbg.fr/viz-bin/qcat?]/A+A/592/A94
}

black hole (SMBH). Mergers are often suggested to perform this task. However, large time delays are often seen between the merger/accretion event and the onset of the radio source (e.g. Emonts et al 2006; Struve et al. 2010a; Shulevski et al. 2012; Maccagni et al. 2014). This seems to rule out a direct link between the two processes and indirect links should instead be considered (see, e.g., Wild et al. 2010). An alternative is the fuelling by gas clouds condensed from the hot halo, or from an available reservoir of cold gas near the centre, as has been suggested by a number of authors (e.g. Allen et al. 2006; Hardcastle et al. 2007; Gaspari et al. 2015). Evidence of such clouds in the process of fuelling the central SMBH has been found in the case of the young radio source PKS 1718-63 (Maccagni et al. 2014, 2016). Finally, the combination with secular processes is also likely to play a role.

Here we present the results of deep radio continuum and H I observations of the nearby, low radio power and gas-rich early-type galaxy, NGC 3998. The observations reveal a particularly intriguing and uncommon structure of the radio continuum emission. The presence of the H I-rich structure allows to explore the connection between the radio lobes and the large reservoir of gas in the system.

\section{Overview of NGC 3998}

NGC 3998 is a nearby ${ }^{1}$ S0 galaxy located at the outskirts of the Ursa Major group and recently studied in detail as part of

\footnotetext{
1 In this paper we assume a distance of $13.7 \mathrm{Mpc}$ for NGC 3998 (Cappellari et al 2011a). At this distance, $1^{\prime \prime}$ corresponds to $65.9 \mathrm{pc}$.
} 
the multi-wavelength ATLAS ${ }^{3 \mathrm{D}}$ survey (Cappellari et al 2011a). This galaxy hosts a low-power, $\sim 10^{22} \mathrm{~W} \mathrm{~Hz}$, radio AGN (Hummel 1980; Wrobel \& Heeschen 1984; Wrobel 1991) with a flat spectrum radio core (Hummel 1980; Hummel et al. 1984; Kharb et al. 2012). Very long baseline interferometry (VLBI) observations have revealed a jet-like structure on the northern side of the nucleus of NGC 3998 (Filho et al. 2002; Helmboldt 2007). The radio core is known to be variable (see also Sect. 3.1) and NGC 3998 also shows variability in the ultraviolet (Maoz et al. 2005) and X-ray (Younes et al. 2012; Hernández-García et al. 2013) regimes. At X-ray energies, NGC 3998 contains a nuclear source with $L_{\mathrm{X}}(2-10 \mathrm{keV}) \sim 2.5 \times 10^{41} \mathrm{erg} \mathrm{s}^{-1}$ (Terashima et al. 2000; Ptak et al. 2004).

The optical spectrum of NGC 3998 shows broad $\mathrm{H} \alpha$ emission lines (Heckman 1980; Devereux 2011) although it is formally classified as a LINER 1.9 (Ho et al. 1997). Imaging studies of the ionised gas in this galaxy have revealed a small $\mathrm{H} \alpha$ disk of about $100 \mathrm{pc}$ (Pogge et al. 2000) surrounded by a larger $(\sim 5 \mathrm{kpc})$, warped structure (Ford et al. 1986; SánchezGallego et al. 2012). The nuclear region of NGC 3998 is also known to contain dust with an estimated mass of $M_{\text {dust }} \sim 5 \times$ $10^{5} M_{\odot}$ (Martini et al. 2013). In terms of its stellar population, NGC 3998 is a red galaxy $(u-r)=4.46$ (SDSS DR6, AdelmanMcCarthy et al. 2008) dominated by evolved stars (McDermid et al. 2015). The single-stellar-population age inside one effective radius $\left(\sim 20^{\prime \prime}\right)$ reported in McDermid et al. (2015) is $11.48 \pm$ 2.00 Gyr. However, young stars (with ages of about 1-10 Myr) may dominate the nuclear $\left(<1^{\prime \prime}\right)$ region of NGC 3998 (González Delgado et al. 2004; González-Martín et al. 2009; Mason et al. 2015).

NGC 3998 contains a large, spatially extended reservoir of HI arranged in a disk-like structure (Knapp et al. 1985). More recently, Serra et al. (2012) showed that the H I disk is many times larger than the optical component and has a mass of $2.8 \times 10^{8} M_{\odot}$. Studies of the H I content of galaxies in Ursa Major (Pak et al. 2014, and references therein) have noted the presence of tidal gas around NGC 3998, which is also seen in the Serra et al. (2012) results. This is likely connected to the fact that NGC 3998 is located in a region of fairly high galaxy density (Cappellari et al. 2011b). CO observations by Baldi et al. (2015b) revealed that NGC 3998 hosts molecular gas with an $\mathrm{H}_{2}$ mass of $\sim 1.7 \times 10^{7} M_{\odot}$.

\section{Observations and data reduction}

We conducted two campaigns to observe NGC 3998 with the Westerbork Synthesis Radio Telescope (WSRT). The observations performed with a relatively narrow (spectral line) band were focused on the study of the $\mathrm{H}$ I, but have also provided highquality radio continuum images. In 2015, we further conducted broad-band observations at wavelengths of $21-\mathrm{cm}$ and $6-\mathrm{cm}$ to better estimate the flux variation of the radio continuum. Below we provide an overview of the calibration and imaging procedure for each set of observations. For the data reduction of both observations, we have used the MIRIAD (Sault et al. 1995) package to perform the data calibration and imaging.

\subsection{Narrow-band observations}

The spectral line observations were conducted as a deep followup to those presented in Serra et al. (2012) which were part of the ATLAS $^{3 \mathrm{D}}$ survey. We obtained nine 12-hour tracks during the period from 2009 to 2011 and a summary of the observational
Table 1. Summary of WSRT 21-cm narrow-band observations.

\begin{tabular}{ll}
\hline \hline$N_{\text {dishes }}$ & $11-12$ \\
Total observing time & $108 \mathrm{~h}$ \\
Observing period & June 2009 - May 2011 \\
Bandwidth/channels & $20 \mathrm{MHz} / 1024$ \\
Velocity resolution & $4.2 \mathrm{~km} \mathrm{~s}^{-1}$ \\
Continuum resolution & $16.5^{\prime \prime} \times 13.2^{\prime \prime}, \mathrm{PA}=2.4^{\circ}$ \\
$3 \sigma$ noise continuum image & $0.15 \mathrm{mJy} \mathrm{beam}^{-1}$ \\
$3 \sigma$ noise per channel $\left(15^{\prime \prime}\right.$ cube $)$ & $0.13 \mathrm{mJy} \mathrm{beam}^{-1}$ \\
\hline
\end{tabular}

parameters is given in Table 1 . The number of dishes that were available during this period ranged between 11 and 12 . This was due to the permanent absence of one dish to the DIGESTIF project (van Cappellen et al. 2009), and the mechanical maintenance of several other dishes in preparation for APERTIF (Oosterloo et al. 2009). Doppler tracking on a heliocentric velocity of $1000 \mathrm{~km} \mathrm{~s}^{-1}$ was used during the observations. The bandwidth of each observation was $20 \mathrm{MHz}$, distributed over 1024 channels giving a channel width of $4.2 \mathrm{~km} \mathrm{~s}^{-1}$. In making the datacubes, the data were binned to a velocity resolution of $8.4 \mathrm{~km} \mathrm{~s}^{-1}$.

The initial part of the calibration followed the standard steps such as flux and bandpass calibration. We then inspected the target spectrum and identified which channels were free of line emission. We then created line and continuum visibility datasets by doing a continuum subtraction, using a fourth-order polynomial to model the bandpass over the line-free channels. We performed phase-only self-calibration on the continuum dataset and the calibration tables derived in this way were also copied to the line dataset. This process produced continuum images which were dynamic range limited due to direction-dependent errors around a bright continuum source at the edge of the field of view. Such direction dependent errors are due to a combination of small pointing errors and the effects of standing waves in the optics of the WSRT dishes. We solved this issue by doing a direction dependent calibration known as "peeling".

The spectral line images were made using the Robust weighting scheme (Briggs 1995) with a robustness of 0.4 and using three different tapering schemes, corresponding to spatial resolutions of $15^{\prime \prime}, 30^{\prime \prime}$ and $60^{\prime \prime}$. We then cleaned each cube by the iterative use of threshold masking (on the cleaned and residual images). The high-resolution cubes we use for examining the kinematics of the $\mathrm{HI}$, while the low-resolution cubes we use for studying the properties of the extended, low surface brightness gas.

We then imaged the combined continuum visibility data using uniform weighting and cleaned the continuum image using a similar method of iterative masking and cleaning that we used to image the line data.

\subsection{Broad-band observations}

The second observing campaign comprised follow-up radio continuum observations at $21-\mathrm{cm}$ and $6-\mathrm{cm}$ in June 2015 . The relevant information for the broad-band observations are summarized in Table 2. The purpose of these observations was to do broad-band measurements of the variable radio continuum

\footnotetext{
2 http://www.astron.nl/ oosterlo/peeling.pdf
} 
Table 2. Summary of WSRT broad-band observations.

\begin{tabular}{ll}
\hline \hline$N_{\text {dishes }}$ & 5 \\
Observing time per track & $12 \mathrm{~h}$ \\
Subbands/bandwidth/channels & $8 \times 20 \mathrm{MHz} / 128$ \\
\hline 21-cm observations & \\
Date observed & 2 June 2015 \\
Band centres [MHz] & $1450,1432,1410,1392$, \\
& $1370,1350,1330,1311$ \\
$3 \sigma$ noise continuum image & $0.43 \mathrm{mJy} \mathrm{beam}^{-1}$ \\
Continuum resolution & $16.5^{\prime \prime} \times 12.1^{\prime \prime}, \mathrm{PA}=85.8^{\circ}$ \\
\hline 6-cm observations & \\
Date observed & $20 \mathrm{June} 2015$ \\
Band centres [MHz] & $4858,4876,4894,4912$, \\
& $4930,4948,4966,4984$ \\
$3 \sigma$ noise continuum image & $0.41 \mathrm{mJy} \mathrm{beam^{-1 }}$ \\
Continuum resolution & $7.00^{\prime \prime} \times 4.966^{\prime \prime}, \mathrm{PA}=52.2^{\circ}$ \\
\hline
\end{tabular}

emission of the AGN. Two 12-h tracks were conducted at 6- and 21-cm, respectively. Only five WSRT dishes were available for these observations due to the ongoing upgrade of the WSRT to the phased-array-feed based APERTIF system. For each observation we used the standard $160 \mathrm{MHz}$ bandwidth setups available with the WSRT.

Each of the $20 \mathrm{MHz}$ sub-bands were covered by 128 channels in dual polarization. Standard flux and bandpass corrections were derived from short observations of 3C 48 and were copied to the target visibilities. We then performed iterative selfcalibration on each sub-band in the same way as described in the previous section. The final images were produced using uniform weighting.

\section{Results}

\subsection{The radio continuum}

In Fig. 1 we present the radio continuum image of NGC 3998 as derived from the narrow-band observations. As indicated in Table 2 and the previous section, less than half of the WSRT array was available for the broad-band observations conducted in 2015. As a result, the $6-\mathrm{cm}$ observations are not sensitive to any diffuse, faint structure, and we only detect the unresolved core. Similarly, the broad-band observations at $21-\mathrm{cm}$ are less sensitive than the narrow-band observations. Assuming the flux density varies as a function of frequency $S \propto v^{-\alpha}$, we calculate a spectral index $\alpha$ from the 21- and 6-cm broad-band observations for the core of 0.19 , which is flat and characteristic of synchrotron self-absorption commonly associated with a radio AGN. The rest of the analysis and discussion in this paper focuses on the $21-\mathrm{cm}$ narrow-band emission, unless otherwise explicitly stated.

The narrow-band continuum emission appears particularly interesting and is much more extended than was previously known. The new observations reveal two poorly collimated, lowsurface brightness structures extending $\mathrm{N}$ and $\mathrm{S}$ of the core. The projected linear extent of each of these lobes is $\sim 10 \mathrm{kpc}$. A hint for the presence of the southern (and brighter) lobe is visible on the NRAO VLA Sky Survey (NVSS, Condon et al. 1998) images and was also earlier reported by Wrobel \& Heeschen (1984) and Knapp et al. (1985).

The "S-shaped" large-scale morphology of these lobes is immediately apparent from Fig. 1. Interestingly, the shape of the radio continuum appears to mirror the warped structure of the

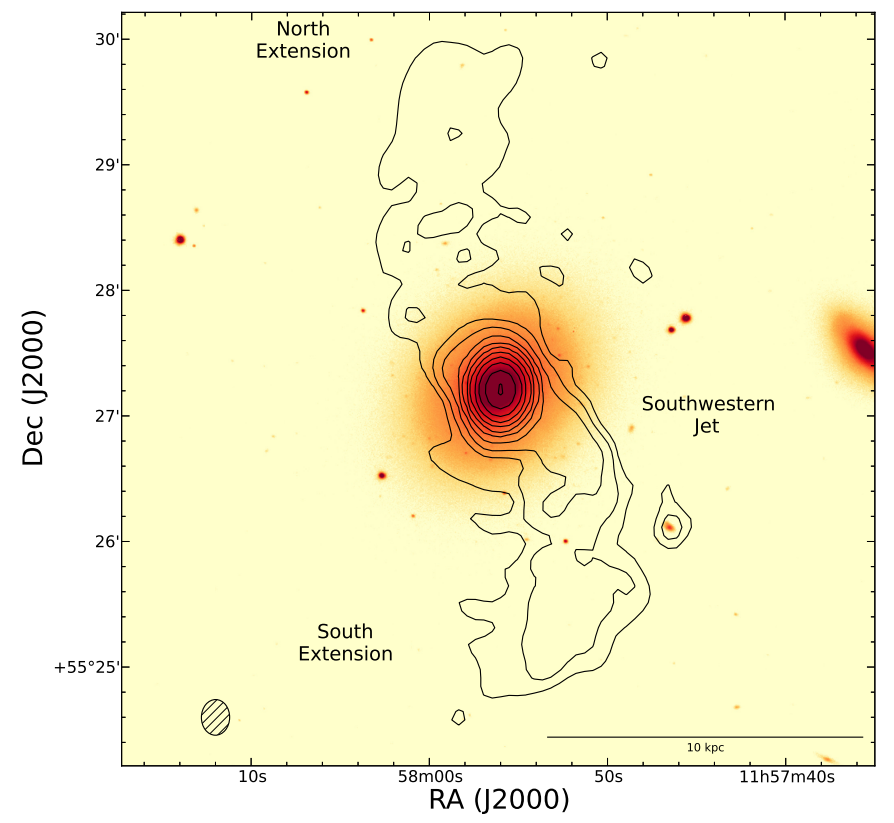

Fig. 1. Radio continuum contours from the narrow-band 21-cm of NGC 3998 overlaid on an $r$-band optical image from the Sloan Digital Sky Survey (Alam et al. 2015). The contours from the radio continuum were produced from the $21-\mathrm{cm}$ narrow-band data using uniform weighting - which corresponds to a robustness parameter in MIRIAD of -2 . The beam-size is indicated in the lower-left corner of the image. The contours are given by $S_{\mathrm{c}}=S_{0} \times 2^{n} \mathrm{mJy} \mathrm{beam}^{-1}$, for $n=0,1,2, \ldots, 10$, where $S_{0}=0.150 \mathrm{mJy} \mathrm{beam}^{-1}$ is the $3 \sigma$ sensitivity as indicated in Table 1.

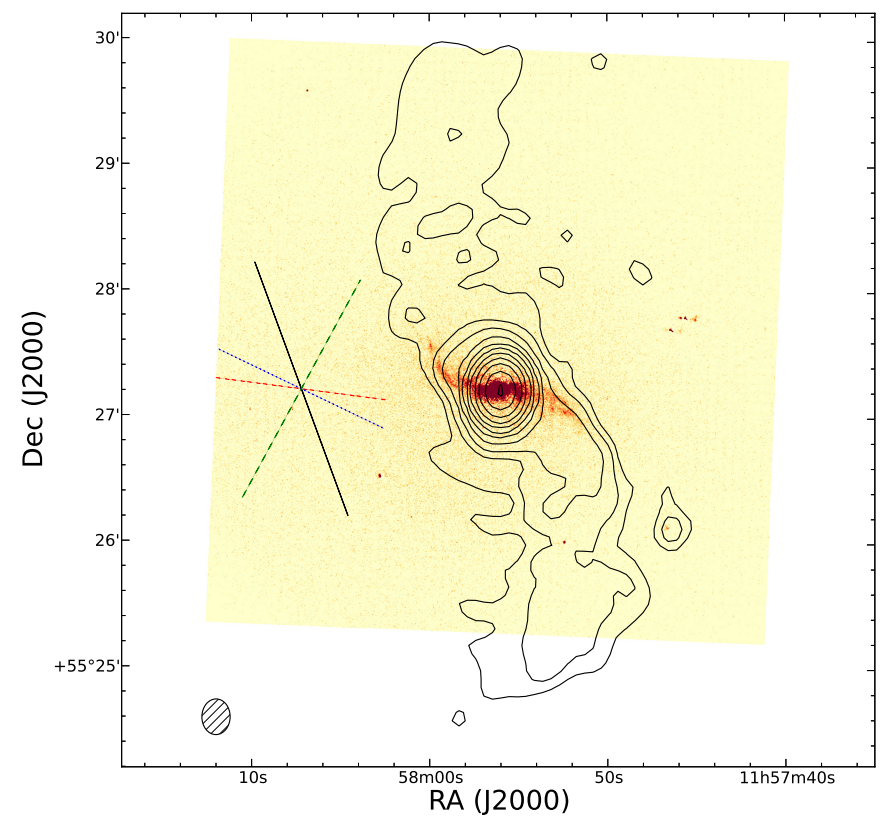

Fig. 2. Radio continuum contours from the narrow-band $21-\mathrm{cm}$ of NGC 3998 overlaid on the continuum-subtracted $\mathrm{H} \alpha$ image from Sánchez-Gallego et al. (2012). The contours are described in Fig. 1. On the left we show a depiction of the position angles of the various galaxy components. Green-dashed: stars $\left(\mathrm{PA} \sim 135^{\circ}\right.$, from Krajnović et al. 2011), black-solid: inner $\sim 5 \mathrm{kpc}$ of radio continuum (PA $\sim 33^{\circ}$ ), blue-dotted: $\mathrm{H}$ I disk $\left(\mathrm{PA} \sim 65^{\circ}\right)$, red-dot-dash: $\mathrm{H} \alpha$ disk $\left(\mathrm{PA} \sim 84^{\circ}\right)$.

ionised gas disk (Sánchez-Gallego et al. 2012) as illustrated in Fig. 2. We discuss this in more detail below. In the central region, we observe a bright core while the brighter part of the SW jet 
Table 3. Flux density and radio luminosity for the continuum features denoted in Fig. 1.

\begin{tabular}{lrc}
\hline \hline Continuum feature & \multicolumn{1}{c}{\begin{tabular}{c}
\multicolumn{1}{c}{$\begin{array}{c}1.4 \mathrm{GHz} \\
\mathrm{mJy}\end{array}$} \\
\end{tabular}} & $\begin{array}{c}L_{1.4 \mathrm{GHz}} \\
10^{31} \mathrm{erg} \mathrm{s}^{-1}\end{array}$ \\
\hline Total & $195.6 \pm 10.3$ & 61.5 \\
Core & $176.7 \pm 8.9$ & 55.6 \\
Northern extension & $7.1 \pm 1.5$ & 2.2 \\
Southern extension & $11.6 \pm 1.4$ & 3.6 \\
Southwestern jet & $3.3 \pm 0.3$ & 1.1 \\
\hline
\end{tabular}

structure extends to $\sim 1.5 \mathrm{kpc}$ from the nucleus. Interestingly, the VLBI (Very Long Baseline Array, VLBA, and European VLBI Network, EVN) observations reported by Helmboldt (2007) and Filho et al. (2002) show an extension on the scale of $\sim 10$ mas to the north of the core. The connection between the mas and the arcsec scale will need to be investigated with new observations.

We measured integrated flux densities from the radio continuum map using the Viewer from the Common Astronomy Software Applications (CASA) ${ }^{3}$ by drawing a polygon around the region of interest; each polygon large enough to encompass emission brighter than approximately $3 \sigma$ as indicated in Table 1. For the core emission we fitted a Gaussian shape to the unresolved emission.

Assuming a $5 \%$ uncertainty in the absolute flux scale at WSRT, we calculated the total error as the quadratic sum of the theoretical uncertainty over the region of interest and the systematic uncertainty associated with the measured flux.

The integrated flux densities, errors and the associated luminosities are presented in Table 3. The integrated flux density of the northern lobe is $7.1 \mathrm{mJy}$ while the southern lobe has a slightly higher flux for the extended part (11.6 mJy). For the bright, linear extension directly to the southwest of the core we derive a flux density of approximately $3.3 \mathrm{mJy}$.

In addition to the S-shape of the continuum emission, two other interesting properties can be noted: the relatively low surface brightness of the extended structures and the large core dominance. The newly discovered lobes have a surface brightness of about $5 \mathrm{mJy} \operatorname{arcmin}^{-2}$. This is low and at the limit of what is detected with currently available surveys and indeed only the southern lobe of NGC 3998 is barely visible in the NVSS image. Structures with low surface brightness radio continuum emission are often considered to be a signature of remnant-like emission, i.e. emission from lobes that are in the process of fading away because they are not supplied anymore with fresh radio plasma (e.g. Saripalli et al. 2012; Brienza et al. 2016). Furthermore, the detected radio structure is strongly core dominated. The ratio $S_{\text {core }} / S_{\text {ext }} \sim 8$ is much higher than what is typically found for FRIs (Morganti et al. 1997; Laing \& Bridle 2014) and is almost an order of magnitude higher than in even the radio sources of even lower power considered by Baldi et al. (2015a). We will return to these points later in the discussion.

Based on the narrow-band observations, we measure a radio continuum flux density of $176.7 \mathrm{mJy}$ for the core emission. This value is much higher than measured from the NVSS image (106.8 mJy) and by Knapp et al. (1985), 100 mJy. In order to check whether our measurement could be due to an error in the flux calibration, we compared our measurements of the flux densities of other sources in the field with those in the NVSS image. We found the fluxes measured from our images to be within 5\% of the values measured from the NVSS image.

\footnotetext{
3 http://casa.nrao.edu
}

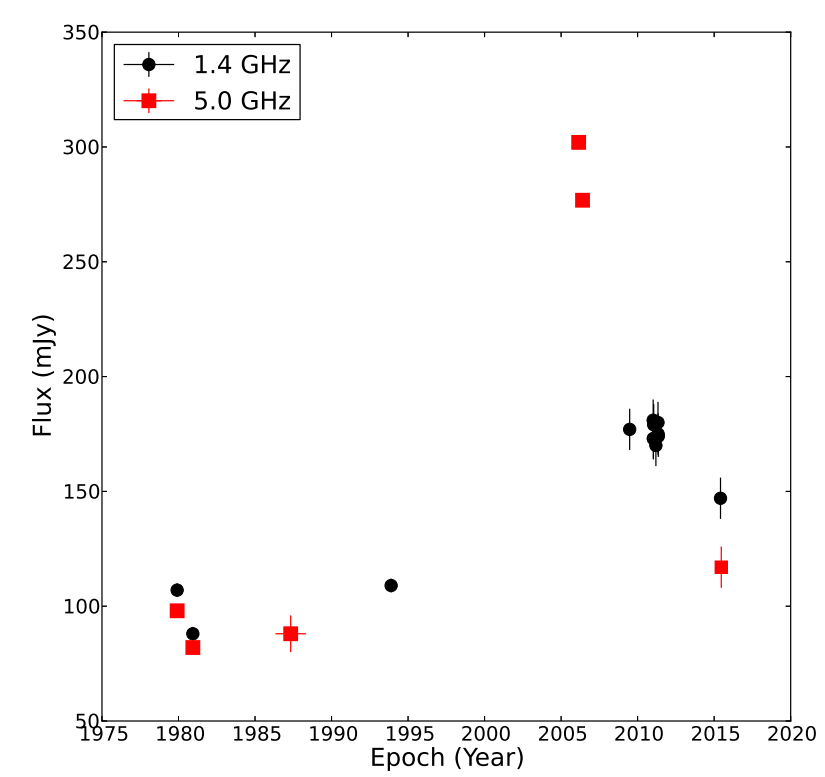

Fig. 3. Variability of the radio continuum emission from the core of NGC 3998. The red squares correspond to measurements at $6-\mathrm{cm}$, the black circles at $21-\mathrm{cm}$. The epochs, measurements and associated uncertainties (where available) are presented in Table 4.

Although the radio core of NGC 3998 is known to be variable, the flux density of NGC 3998 did not show any time variation for the duration of the narrow-band campaign, i.e., the flux density of the core remained constant from 2009 to 2011. However, when considered over a longer time span, it is clear that the radio core varies substantially in flux density. In Fig. $3^{4}$ we compare our measurements with those from the literature as a function of time. We also list the measurements used to construct this plot in Table 4, with the associated uncertainties where available. The flux reported from the 2006 VLBA observations at 6-cm of Helmboldt (2007) is $276.8 \mathrm{mJy}$, suggesting that our observations may have been during a phase of decreasing flux after a flare. This is consistent with our follow-up broadband radio continuum observations of NGC 3998 conducted during 2015, which have yielded a flux density for the core emission of $148 \mathrm{mJy}$ at $21-\mathrm{cm}$ and $117 \mathrm{mJy}$ at $6-\mathrm{cm}$. Kharb et al. (2012) reported their 2006 measurement of the $4.9 \mathrm{GHz}$ flux density of $302 \mathrm{mJy}$ which is more than three times higher than the measurement presented in Wrobel (1991) of $83 \mathrm{mJy}$. Gregory et al. (1996) presented a $4.85 \mathrm{GHz}$ flux density of $88 \mathrm{mJy}$, as measured with the Green Bank telescope during the observational campaign in 1986 . We measure a $5 \mathrm{GHz}$ flux density of $117 \mathrm{mJy}$. Thus, also at this higher frequency, the core flux of NGC 3998 appears to be declining after a recent burst.

\subsection{Atomic gas in NGC 3998}

In Fig. 4 we present the HI column density map and velocity field derived from the $30^{\prime \prime}$ image cube and the major axis position-velocity (PV) diagram from the $15^{\prime \prime}$ data. The most striking feature of the H I in NGC 3998 is that much of the H I is arranged in a highly inclined disk that is roughly polar with respect to the distribution of the stars while at larger radii the H I morphology is less regular. In Fig. 5 we present an image of

\footnotetext{
4 These measurements were derived using different telescopes, with different resolutions/UV-coverages. We expect that this will have a small effect on the observed variability depicted in the figure.
} 

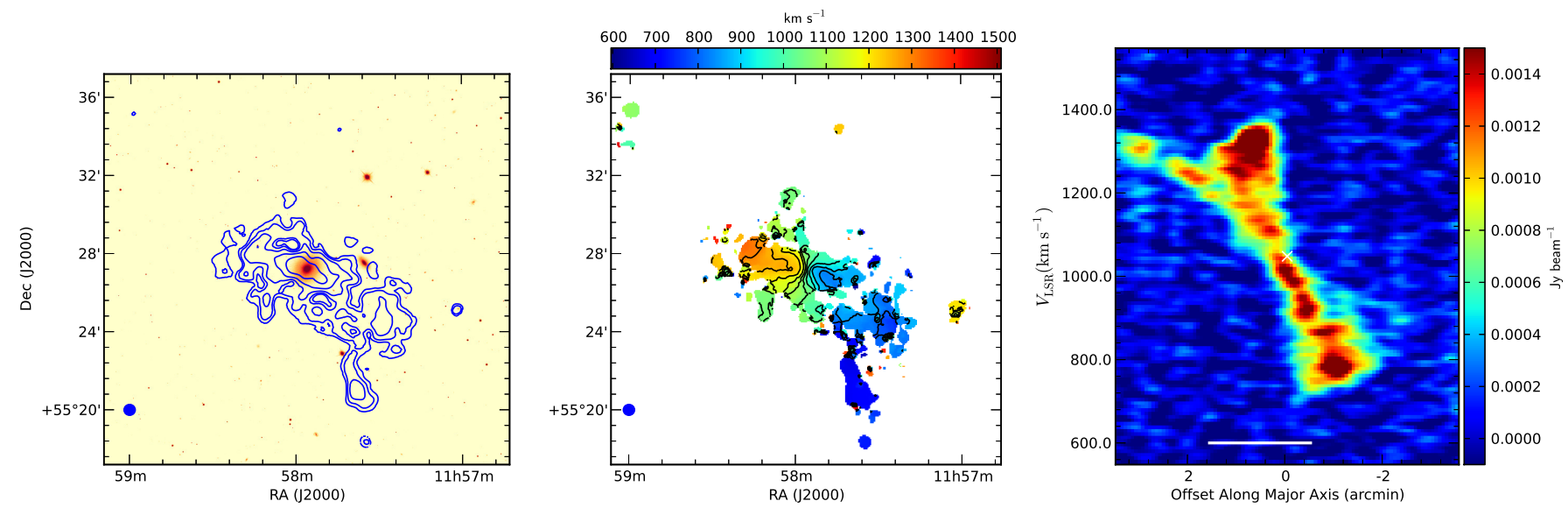

Fig. 4. Left: H I column density contours, calculated from the $30^{\prime \prime}$ cube, overlaid on the $r$-band SDSS image. The contours are given by $S_{\mathrm{HI}}=$ $2^{n} \times 10^{19} \mathrm{~cm}^{-2}(n=0,1,2, \ldots)$. The beam size is indicated in the lower-left corner of the figure. Middle: intensity-weighted mean velocity field calculated from the $30^{\prime \prime}$ cube, the iso-velocity contours correspond to $V_{\text {sys }} \pm n \times 50 \mathrm{~km} \mathrm{~s}^{-1}$, where $n=1,2,3, \ldots$ and $V_{\text {sys }}=1048 \mathrm{~km} \mathrm{~s}^{-1}$. Right: position-velocity diagram along the major axis of the $15^{\prime \prime} \mathrm{H}$ I cube. The white scale bar indicates $10 \mathrm{kpc}$.

Table 4. Flux variability of NGC 3998.

\begin{tabular}{llrc}
\hline \hline Date & $\begin{array}{l}\text { Frequency } \\
\text { MHz }\end{array}$ & $\begin{array}{r}\text { Flux density } \\
\text { mJy }\end{array}$ & Reference \\
\hline $30 / 11 / 1979^{a}$ & 1500 & $107 \pm 3$ & $(1)$ \\
$30 / 11 / 1979^{a}$ & 4900 & $98 \pm 3$ & $(1)$ \\
$09 / 12 / 1980$ & 1500 & $98 \pm 3$ & $(1)$ \\
$09 / 12 / 1980$ & 4900 & $83 \pm 3$ & $(1)$ \\
$02 / 05 / 1987^{a}$ & 4850 & $88 \pm 8$ & $(2)$ \\
$23 / 11 / 1993$ & 1420 & $109 \pm 3$ & $(3)$ \\
$24 / 02 / 2006$ & 4860 & 302 & $(4)$ \\
$27 / 05 / 2006$ & 4844 & 277 & $(5)$ \\
$25 / 06 / 2009$ & 1420 & $177 \pm 9$ & This work. \\
$15 / 02 / 2011^{b}$ & 1420 & $175 \pm 9$ & This work. \\
$02 / 06 / 2015$ & 1381 & $149 \pm 7$ & This work. \\
$20 / 06 / 2015$ & 4901 & $118 \pm 6$ & This work. \\
\hline
\end{tabular}

Notes. We present the epoch, frequency, flux density and uncertainty (where available) and the associated reference for the measurement. (a) In cases where there was an ambiguity in the date (e.g., survey data/several epochs), the median date was used. ${ }^{(b)}$ We show the median date and flux density deduced from our 2011 observations.

References. (1) Wrobel \& Heeschen (1984); (2) Gregory et al. (1996); (3) Condon et al. (1998); (4) Kharb et al. (2012); (5) Helmboldt (2007).

the HI emission in NGC 3998 and the neighbouring galaxies. The emission in NGC 3998 is lopsided and low-column density gas extends to the S-W - towards NGC 3982 and NGC 3972. Additionally, there are streams and trails of low column density gas which indicates tidal interactions between the three most massive galaxies in this group. The structure of the H I could suggest that galaxy interactions have occurred and that (some of) the H I gas found in NGC 3998 originates from tidal interactions with other members of the group, similar to the distribution observed around M 81 (Yun et al. 1994; also see Haynes et al. 1984 and Hibbard et al. 2001 for more examples of interacting systems).

NGC 3998 is part of a group of galaxies at the edge of the Ursa Major cluster (Tully et al. 1996), several of which are detected by our observations (Fig. 5). The $5 \sigma$ detection limit of our observations is about $5 \times 10^{5} M_{\odot}$ for a velocity width of $8.4 \mathrm{~km} \mathrm{~s}^{-1}$. In Table 5 we provide the integrated flux densities, the associated uncertainties and the H I masses for each of the detections, as measured using the $30^{\prime \prime}$ cube, which was corrected
Table 5. Integrated flux densities and H I masses for the galaxies detected in our observations.

\begin{tabular}{lrc}
\hline \hline Galaxy & $\begin{array}{r}\text { Integrated flux density } \\
\mathrm{Jy} \mathrm{km} \mathrm{s}^{-1}\end{array}$ & $\begin{array}{c}\mathrm{H} \mathrm{I} \text { Mass } \\
\log M_{\mathrm{HI}} / M_{\odot}\end{array}$ \\
\hline NGC 3998 & $9.83 \pm 0.13$ & 8.63 \\
NGC 3972 & $15.96 \pm 0.08$ & 8.81 \\
UGC 6988 & $1.23 \pm 0.19$ & 7.74 \\
UGC 6919 & $0.76 \pm 0.23$ & 7.52 \\
SDSSJ115701 & $0.05 \pm 0.03$ & 6.36 \\
NGC 3982 & $23.31 \pm 0.29$ & 9.01 \\
Blob & $0.41 \pm 0.15$ & 7.26 \\
\hline
\end{tabular}

for the primary beam. When calculating the H I for each object we used the distance to NGC 3998 of $13.7 \mathrm{Mpc}$, which fits in with our interpretation of these objects being involved in intergroup interactions. This will be discussed in the Sections below.

From our data we derive a total H I mass of the H I disk in NGC 3998 of $4.3 \times 10^{8} M_{\odot}$, which is considerably higher than the value reported by Serra et al. (2012). This is likely due to the increased detection of low column density $\mathrm{H}$ I in our deeper observations. The H I mass determined for NGC 3982 is close to the value mentioned in Boselli et al. (2014), and the H I mass for NGC 3972 is similar to the value mentioned in Sanders \& Verheijen (1998). SDSSJ115701 is the only other SDSS object for which we have a clear H I detection.

The velocity field in Fig. 4 shows a well-defined gradient along the major axis of the H I disk, suggesting that its kinematics are dominated by regular rotation, at least for radii $<10 \mathrm{kpc}$. The scale-bar $(10 \mathrm{kpc})$ in the PV diagram indicates the approximate extent of the regular rotation. Beyond this radius, the H I structure becomes more filamentary. The spectra at some locations with radii larger than $10 \mathrm{kpc}$ show multiple components, suggesting that this gas may still be settling into a disk. At even larger radii, some clouds without any obvious optical counterpart are found, the largest of which is found about 11' north of NGC 3982 (and named "Blob" in Table 5). In addition, the $\mathrm{H}$ I distribution and kinematics in the outer regions of NGC 3982 are quite disturbed.

Faint structures and possible streams have been observed with the MegaCaM instrument on the Canada-France-Hawaii Telescope, as part of the CFHT Large Programme Mass Assembly of Early-Type Galaxies with their Fine Structures 


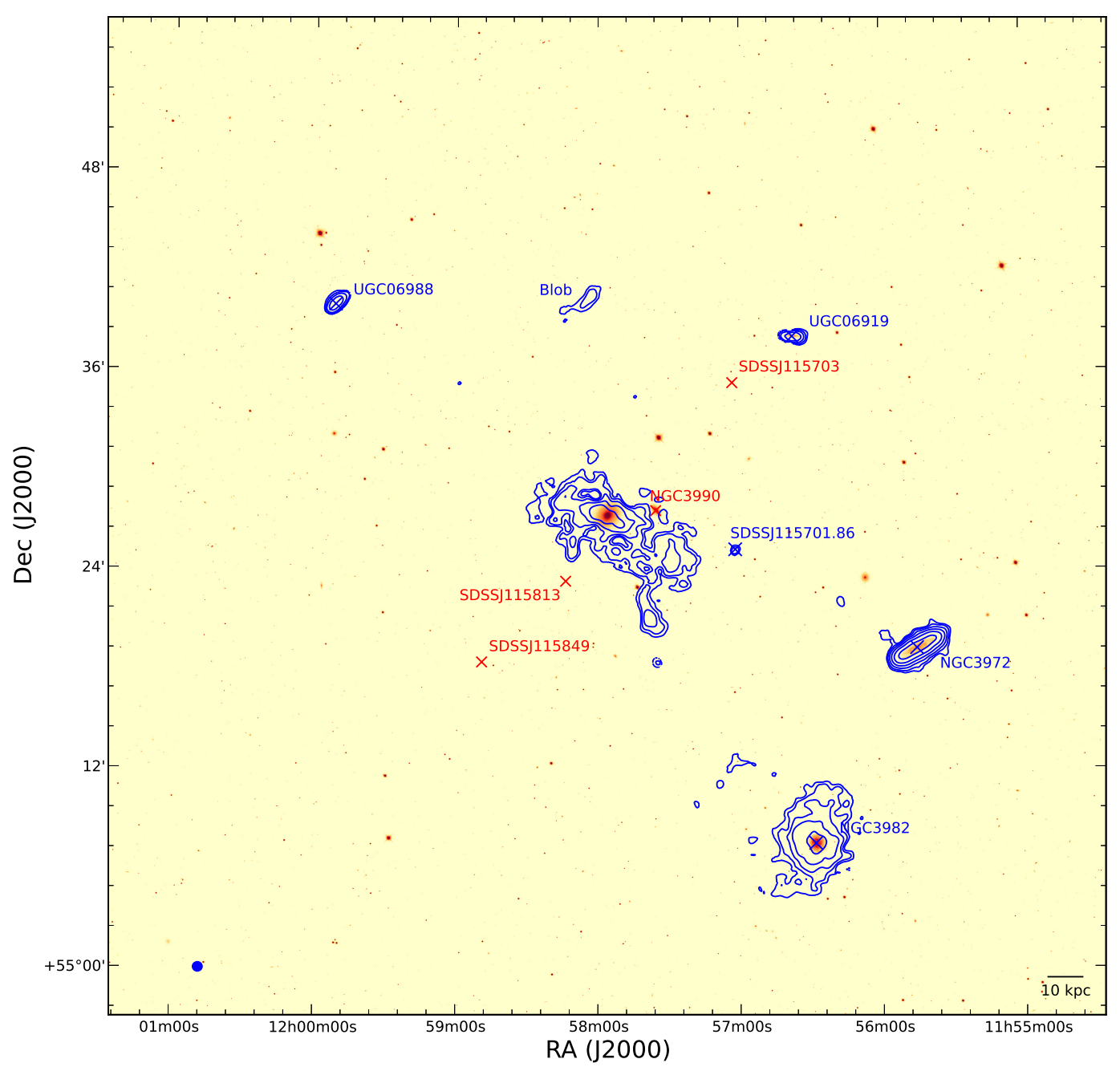

Fig. 5. H I column density contours of NGC 3998 and its neighbours, overlaid on the $r$-band SDSS image. The contours are given by $S_{\mathrm{HI}}=$ $2^{n} \times 10^{19} \mathrm{~cm}^{-2}(n=0,1,2, \ldots)$; the lowest contour corresponds to the detection limit of our observations. The figure is annotated such that blue crosses and labels indicate galaxies with an $\mathrm{H}$ I detection in our work, while a red cross and labels indicates galaxies that do not have an associated detection.

$(\text { MATLAS })^{5}$, which is an extension to the study presented in Duc et al. (2015). However, the detection is limited by the presence of nearby bright stars in the field. Additionally, there is no prominent optical counterpart to the H I structure. Thus, a picture where the H I of NGC 3998 was brought in by a small satellite and is now in the process of settling in NGC 3998 appears a likely scenario. We will come back to this point in Sect. 6 .

It is worth noting that the $\mathrm{H}$ I and the stellar components are arranged in a nearly polar configuration (see also Knapp et al. 1985). A perfectly polar H I configuration can be relatively stable within an oblate mass distribution (which NGC 3998 is likely to have, see Cappellari et al. 2013). Using the available data on the inclination of the H I (den Heijer et al. 2015) and of the optical body (Krajnović et al. 2011; Cappellari et al. 2013) we find that the position angle between the H I and the symmetry plane of the stellar distribution is $80^{\circ}$, but given the uncertainties, a perfectly polar configuration cannot be entirely excluded. In Fig. 2 a sketch of the orientation of the various galaxy components is presented. The angle (measured from North counterclockwise) between the stellar mid-plane and the $\mathrm{HI}$ disc is $\sim 70^{\circ}$, (taking

\footnotetext{
5 http://irfu.cea.fr/Projets/matlas/atlas3D/NGC3998. html
}

the $\mathrm{PA}(\mathrm{star})=135^{\circ}$ as derived from both photometric and kinematic information, Krajnović et al. 2011, and the $\mathrm{PA}(\mathrm{HI})=65^{\circ}$ measured in this work). The morphology of the inner $\mathrm{H} \alpha$ disk strongly suggests that it has a warped structure with the inner parts somewhat more aligned with the stellar body. To further study this, we have made a velocity field of the HI in the inner regions using the high-resolution $\mathrm{H}$ I data cube where we have derived the velocities using Gauss-Hermite fits in order to take into account the slight asymmetric shape of many of the H I profiles in this region (Fig. 6). This velocity field shows that in the very inner region, the $\mathrm{H}$ I disk seems to follow the warped $\mathrm{H} \alpha$ structure. This is visible from the curved kinematical major axis of the velocity field, and from the fact that the kinematical minorand major-axes are not perpendicular. This suggests that differential precession due to torques on the overall gas structure is occurring, in particular in the inner regions. We will discuss this point in Sect. 6.

\section{The nature of the radio continuum structure}

The interesting new finding from our observations is the detection of extended low surface-brightness radio lobes representing relatively rare structures in low power radio sources like 


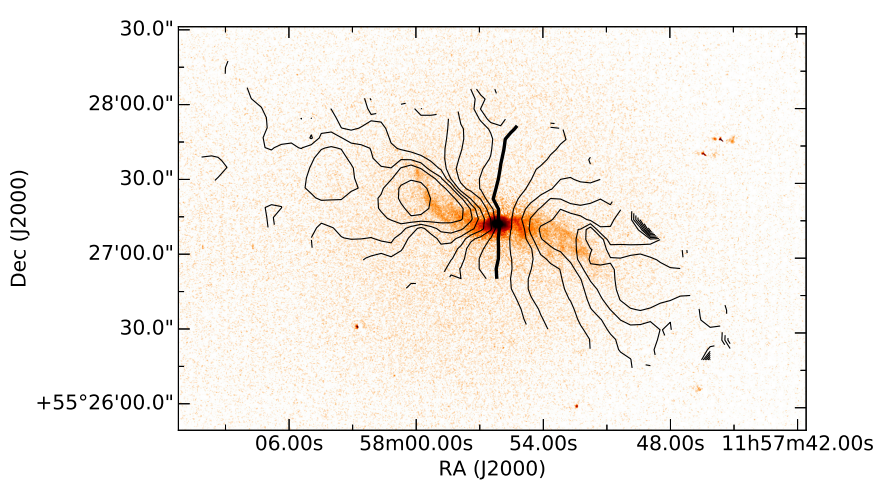

Fig. 6. Velocity contours of the inner H I disk, obtained obtained from Gauss-Hermite fits to the 15" resolution cube, superposed to the $\mathrm{H} \alpha$ image from Sánchez-Gallego et al. (2012). Contour values are 800, 840, $880, \ldots, 1400 \mathrm{~km} \mathrm{~s}^{-1}$. The systemic velocity of $1040 \mathrm{~km} \mathrm{~s}^{-1}$ is indicated by the thick line.

NGC 3998. The low surface brightness of the lobes is comparable to known "remnant" lobes (e.g. Saripalli et al. 2012; Brienza et al. 2016), i.e. structures not directly fuelled anymore by an active nucleus. However, from a morphological point of view, the lobes of NGC 3998 have characteristics which suggest that they are still actively fed by the nucleus. An example of this is in the southern part which shows a collimated kpc-scale jet that may be still feeding the southern lobe.

The study of the ATLAS ${ }^{3 \mathrm{D}}$ sample of nearby early-type galaxies (of which NGC 3998 is part) suggests that this type of radio structure are genuinely rare in low power radio sources. Of the 260 nearby $(D<40 \mathrm{Mpc})$ early-type galaxies included in the ATLAS $^{3 \mathrm{D}}$ survey (and which have $5 \mathrm{GHz}$ nuclear radio powers typically between $10^{18}$ and $10^{22} \mathrm{~W} \mathrm{~Hz}^{-1}$; Nyland et al. 2016), NGC 3998 is among a minority (smaller than $\sim 10 \%$ ) of systems that are known to harbour extended radio emission at high resolution scales (see Nyland et al. 2016). Furthermore, even in the deep, lower spatial resolution continuum images (which are more sensitive to extended emission) obtained for a subset of these objects from WSRT observations (Morganti et al. in prep), only three objects out of more than 100 observed (Serra et al. 2012) were found to have extended emission on the scale of tens of kpc, one of them being NGC 3998 (the other two NGC 3665 and NGC 5322). In this context, it is not surprising that NGC 3998 hosts one of the most massive black holes (above the 90-th percentile) among the galaxies of the ATLAS ${ }^{3 \mathrm{D}}$ sample and that the radio source is among the most powerful of this sample of nearby early-type galaxies (Nyland et al. 2016).

The case of NGC 3998 suggests that the reasons these structures are rare are connected to their properties. Most of the characteristics of the radio continuum structure of NGC 3998 can be explained as being the result of poorly collimated jets. Low power radio sources such as NGC $3998\left(\log P_{1.4 \mathrm{GHz}}=\right.$ $21.63 \mathrm{~W} \mathrm{~Hz}^{-1}$, i.e. well below the typical radio power of FRI radio galaxies) are characterised by a low speed of their plasma flows. Although even in these low power objects the jets generally seem to start with high (relativistic) velocities, they decelerate very early in their evolution, well inside the inner kpc (Laing \& Bridle 2014). Thus, these jets will be dominated by turbulence and by entrainment of the external medium. This results in a high ratio of non-radiating to radiating particles (see e.g. Croston et al. 2008; O'Sullivan et al. 2013; Perucho et al. 2014; Laing \& Bridle 2014). The increased thermal component inside the jet would further slow it down. The consequence of this would be low surface brightness structures which would fade away relatively quickly. In addition to this, the core prominence of the radio continuum structure in NGC 3998 is rather extreme.

Previous studies have shown the $\mathrm{H} \alpha$ disk to be regular (de Francesco et al. 2006; Walsh et al. 2012). Thus, there is no evidence for an interaction from the kinematics of the ionised gas. The observed characteristics could be connected to NGC 3998 beginning a new phase of (restarted) nuclear activity. Consequently, the observed variability of the core may indicate that the fuelling occurs through "discrete events", with new structures being formed and ejected as a result. A similar hypothesis has been recently suggested for the case of another nearby galaxy, NGC 660 (Argo et al. 2015). Further investigating this possibility will require a detailed study of the inner kpc involving high-resolution (VLBI) observations.

Finally, it is important to consider the origin of the overall curved S-shape structure of the extended lobes which (unlike the inner structure and the southern jet) appears to be symmetric on the two sides. We favour the scenario in which these curved radio lobe structures are caused by precession, i.e. a change of orientation of the spin axis of the central supermassive black hole, as is seen in many objects, e.g. M 81 (Martí-Vidal et al. 2011) and Cygnus A (Steenbrugge \& Blundell 2007). Steenbrugge \& Blundell (2007) have provided a number of causes that can produce such a phenomenon. In principle, jet axis precession due to a changing SMBH spin (due to a binary massive black hole associated with a galaxy interaction or merger) could also cause the observed continuum morphology. However, this would not necessarily lead to a connection between the warping disk and the $\mathrm{S}$-shaped morphology. Moreover, there is little evidence for a binary SMBH as observed in the [OIII] emission (i.e. absence of a double peak, Devereux 2011) and the VLBI image shows an extension that is likely due to a jet structure (Filho et al. 2002) associated with a single SMBH. Therefore, in the case of NGC 3998 we explore a scenario in which the cause of the precession is due to a misalignment between the gas channeled onto the accretion disk and the angular momentum vector of the SMBH. In Sect. 6 we discuss the possible origin of the warp observed in the gas, and in Sect. 7 we discuss the link between this warp and the triggering and morphology of the radio continuum of NGC 3998.

\section{An accretion event and the evolution of the gas disk}

The total intensity image and the velocity field of NGC 3998 show that the $\mathrm{HI}$ is distributed in a relatively regularly rotating disk with clear signs of unsettled gas in the outer regions. Thus, the $\mathrm{H}$ I structure can be related to an accretion event, e.g. by capturing gas from nearby companions or by a minor merger with a gas-rich satellite. This event has brought $\sim 10^{8} M_{\odot}$ of $\mathrm{H}$ I into NGC 3998 without visibly affecting the optical structure of the galaxy. Small satellite galaxies are known to be gas rich and, in fact, most of their baryonic mass is in H I (e.g., Maddox et al. 2015). Accretion of a late-type satellite galaxy with a stellar mass of $\sim 10^{8} M_{\odot}$ and $M_{\mathrm{HI}} / M_{\text {star }} \sim 1.0$ would be sufficient to explain the observed H I mass of NGC 3998. Cappellari et al. (2013) find the stellar mass of NGC 3998 to be $\sim 10^{11} M_{\odot}$ so the merger would have a mass ratio $\sim 1000: 1$ which would be consistent with the fact that the stellar body is unperturbed.

In NGC 3998 the inner gas disc is misaligned with respect to the outer gas disc and appears to be closer to the stellar mid-plane. This suggests that in the central regions torques due to the distribution of stellar mass are important. The radius of the inner, misaligned gas disc is $\sim 1 \mathrm{kpc}$. The rotation velocity 
is $\sim 250 \mathrm{~km} \mathrm{~s}^{-1}$ (den Heijer et al. 2015) and the orbital time at this radius is $\sim 1.5 \times 10^{6} \mathrm{yr}$. Therefore, we conclude that the period of time during which the gas disc has been changing orientation within the $1 \mathrm{kpc}$ radius is of the order of a few times $10^{6} \mathrm{yr}$.

Hopkins \& Quataert (2011) argue that stellar torques can also produce orbit crossings and shocks in the gas, which causes the gas to lose angular momentum and accrete onto a central black hole. Given the recent revival of the nuclear activity in NGC 3998 this poses the interesting question whether the start of the alignment of the gas disk is connected to the current episode of nuclear activity in NGC 3998.

The warped inner disk fits in a scenario of a minor gaseous merger. van de Voort et al. (2015) have recently presented numerical simulations of gas accretion by a large early-type galaxy in a cosmological setting. The simulations suggest that it is possible that perfect alignment between a large-scale gas disk and the stellar body can take a long time to achieve, i.e. $\gtrsim 1$ Gyr. This is because as long as gas with misaligned angular momentum is being accreted, the overall gas structure will remain misaligned. In models such as those presented by van de Voort et al. (2015), the realignment starts in the inner regions, where the dynamical timescales are shorter. This implies that when realignment sets in, the inner gas disk should become warped first. A situation as described by van de Voort et al. (2015) may be occurring in NGC 3998. The gas structure beyond a disk radius of approximately $\sim 10 \mathrm{kpc}$ is still fairly irregular; this implies that accretion of gas is still occurring. On the other hand, given the extent of that part of the H I structure which appears in fairly regular rotation, the accretion in NGC 3998 must have started about a Gyr ago, assuming it takes several orbital periods for a regular gas disk to form. Due to the effects discussed by van de Voort et al. (2015), the inner disk has started to align only recently.

\section{Gas disk and the radio continuum emission}

In a number of radio galaxies, the presence of large-scale H I structures have allowed us to compare the timescale of the gas accretion/merger with the age of the radio sources (see e.g. B2 0648+27, Emonts et al 2006; Cen A, B2 0258+35, Struve et al. 2010a,b; and PKS 1718-63, Maccagni et al. 2014). In all these cases, a typical age of the radio source of $10^{6}-10^{7} \mathrm{yr}$ is found and a timescale for the accretion/merging of $10^{8}-10^{9} \mathrm{yr}$. This implies an extremely large delay between the start of the accretion and the onset of nuclear activity and this makes it difficult to identify a direct link between the two phenomena.

For NGC 3998 the multi-frequency radio data necessary to derive the age of the radio source are not available. Thus, we have estimated the age of the radio continuum emission by making assumptions about the jet speed and the time needed to build the observed lobes. Even for low-power jets, it has been claimed that their initial velocity is relativistic, or close to. However, these jets are observed to rapidly decelerate to subrelativistic speeds, and this is likely happening well inside the inner few kiloparsecs. The best example is M84 $\left(P_{1.4 \mathrm{GHz}} \sim\right.$ $10^{23.4} \mathrm{~W} \mathrm{~Hz}^{-1}$ ) for which Laing \& Bridle (2014) suggest that the jets are initially relativistic, but the deceleration scale is estimated to be only $1.8 \mathrm{kpc}$. Beyond that radius the velocities must be quite small (i.e. below $c / 10$ ). The lowest-power radio galaxy where the characteristic structure of deceleration from relativistic speeds is observed is B2 1122+39 (Laing et al. 1999).

Direct determination of jet speeds has been done only in a few cases (e.g. NGC 3801 and Cen A; Croston et al. 2007, 2009). In those cases, the values (ranging between 850 and $2600 \mathrm{~km} \mathrm{~s}^{-1}$ respectively) have been derived from the presence of X-ray shells around the radio emission and deriving the Mach number of the radio flow. In NGC 3998, the radio emission is more relaxed so we do not consider the flow to have a supersonic speed (i.e. no shock is observed). Sound speed of the jets have been given in Morganti et al. (1987) for a group of low-power radio galaxies and these range between 700 and $1700 \mathrm{~km} \mathrm{~s}^{-1}$. Thus, for the large-scale continuum structures of NGC 3998 we assume a low jet speed between 1 and $2 \times 10^{3} \mathrm{~km} \mathrm{~s}^{-1}$. We also assume a distance from the centre of the galaxy to the projected edge of the radio continuum emission of $10 \mathrm{kpc}$. The derived timescales $\tau_{\text {jet }}$ are between $5 \times 10^{6}$ and $10^{7} \mathrm{yr}$ and similar to those derived for, e.g., NGC 3801 and the inner lobes of Cen A (Croston et al. 2007, 2009).

As in the other objects mentioned above, the time since the accretion event started is much longer than the age of the radio source. However, we note that our estimate of the age of the radio emission is similar to the timescale we estimated for the inner warp in NGC 3998 to develop. Moreover, we also note the morphological similarity between the warp of the inner gas disk and the S-shaped radio continuum emission (see Fig. 6). Based on this, we suggest that the start of the realignment of the inner gas disk could be indeed an indication of the moment when the gas has reached the central regions. Additionally, the effects of the stellar torques provide the necessary loss of angular momentum which would cause gas clouds to fall toward the SMBH, fuel it and make it active. In this scenario, the connection between the warping of the gas structure and the morphology of the radio lobes would be that the SMBH spin and, therefore, the jet axis are adapting to the changing angular momentum of the accreting gas. Indeed, such jet precession is expected to generate the radio continuum morphology observed in systems such as Mrk 6 (Kharb et al. 2006) and NGC 326 (Ekers et al. 1978).

\section{Energetics and triggering}

Based on its optical emission line ratios, NGC 3998 is classified as a LINER (Ho et al. 1997). As is typical for this type of lowluminosity AGN, the accretion rate of the SMBH in the nucleus of NGC 3998 is believed to be quite low. Eracleous et al. (2010) reported an Eddington ratio of $4 \times 10^{-4}$, indicating inefficient $\mathrm{SMBH}$ accretion well below the Eddington luminosity, consistent with the classification of NGC 3998 as a Low Excitation Radio Galaxy (LERG; Best \& Heckman 2012). The inefficient accretion characteristic of LERGs may be driven by gas cooling from the hot galactic halo supplying fuel to the SMBH via Bondi accretion (Croton et al. 2006; Allen et al. 2006; Hardcastle et al. 2007; Balmaverde et al. 2008), or perhaps by chaotic accretion of turbulent clouds (e.g., Gaspari et al. 2015, and references therein).

Deeper insights into the underlying accretion physics in the nucleus of NGC 3998 may be gained by examining the efficiency of the radio lobes relative to the mass accretion rate. Previous studies have reported jet efficiencies of a few percent based on studies of the correlation between radio jet power and the Bondi mass accretion rate ( $\dot{m}_{\mathrm{B}}$, Bondi 1952) for samples of nearby, inefficiently-accreting radio AGNs (Allen et al. 2006; Balmaverde et al. 2008; Russell et al. 2013; Nemmen \& Tchekhovskoy 2015).

The radio jet power, $P_{\text {jet }}$, may be estimated based on the results of scaling relations between $1.4 \mathrm{GHz}$ radio luminosity and the jet power provided. Using the relation given in Cavagnolo et al. (2010) and the total $1.4 \mathrm{GHz}$ luminosity of NGC 3998 of $6.15 \times 10^{37} \mathrm{erg} \mathrm{s}^{-1}, P_{\text {jet }} \sim 1.64 \times 10^{42} \mathrm{erg} \mathrm{s}^{-1}$. Based on 
the relation between $P_{\text {jet }}$ and the "Bondi power", $P_{\mathrm{B}}=\dot{m}_{\mathrm{B}} c^{2}$ provided in Balmaverde et al. (2008) and our estimate of the jet power in NGC 3998, we estimate a Bondi accretion rate in NGC 3998 of $2 \times 10^{-3} M_{\odot} \mathrm{yr}^{-1}$. This accretion rate ${ }^{6}$ is consistent with that estimated in Devereux (2011) based on the observed $\mathrm{H} \alpha$ and X-ray emission in the NGC 3998 nucleus of $\dot{m} \sim 3.6 \times 10^{-3} M_{\odot} \mathrm{yr}^{-1}$. Thus, about $1 \%$ of the mass inflow rate is converted in jet mechanical energy in NGC 3998, consistent with other low-power radio AGNs (Balmaverde et al. 2008; Nemmen \& Tchekhovskoy 2015).

The presence of clouds either from the cooling of a hot halo or from the accretion of gas is likely quite common in earlytype galaxies (Negri et al. 2015). These galaxies are now recognised to often have a rich ISM and a relatively large reservoir of gas (Mathews \& Brighenti 2003; Morganti et al. 2006; Young et al. 2011; Serra et al. 2012). In fact, the presence of clouds of cold gas in unsettled/chaotic orbits, possibly triggering/fuelling the SMBH and maintaining a galaxy's nuclear activity, has been suggested for other (radio) AGN from, e.g., H I absorption features (e.g. Maccagni et al. 2014, and references therein) and by the high velocity dispersion of the molecular gas in the central regions of some low-luminosity AGN (Mueller Sánchez 2006, 2013; Neumayer et al. 2007; Mezcua et al. 2015; Maccagni et al. 2016).

As mentioned above, it is possible that the torques responsible for realigning the gas disk in the central regions, by which some gas clouds lose their angular momentum and fall into the $\mathrm{SMBH}$, is the mechanism actually responsible for providing discreet "feeding events" for the AGN. This can also explain the variability observed in radio (see Sect. 4.1). Furthermore, we suggest that low-power radio continuum lobes, like the ones we see in NGC 3998, are short-lived structures. This is due to the low speed of the jets and the consequent strong turbulence and entrainment of material from the interstellar/intergalactic medium (ISM/IGM). Thus, with NGC 3998 we have caught a galaxy not too late after the event that fuelling the SMBH has occurred while the radio lobes were still visible.

\section{Summary and conclusions}

We have presented deep radio continuum and $\mathrm{H}$ I observations of the galaxy NGC 3998, performed with the WSRT. The good sensitivity of our observations has revealed two important aspects of NGC 3998 and the surrounding environment. Our conclusions are as follows:

1. The radio continuum observations reveal two lowsurface brightness, S-shaped lobes with a total extent of about $20 \mathrm{kpc}$. In galaxies of similar low radio power as NGC 3998 such structures are only seldom seen. The radio continuum morphology is extremely core dominated $\left(S_{\text {core }} / S_{\text {ext }}=8\right)$ and asymmetric on small scales with a $1.5 \mathrm{kpc}$ jet only on one side. We suggest the radio emission to be the result of poorly collimated jets characterised by a low velocity of the plasma flow. These structures are known to be dominated by turbulence and entrainment of material from the ISM/IGM, which would further slow down the plasma flow. Hence, they will fade away on relatively short timescales, therefore explaining the rarity of low surface brightness radio lobes in galaxies like NGC 3998.

\footnotetext{
6 We emphasize that this is only a rough estimate since the in trinsic scatter in both the $P_{\text {jet }}-P_{\text {radio }}$ and $P_{\text {jet }}-P_{\text {acc }}$ relation are both $\sim 0.7$ dex (Balmaverde et al. 2008; Cavagnolo et al. 2010; Nemmen \& Tchekhovskoy 2015).
}

2. The HI is distributed in a disk which is almost, but possibly not quite, polar with respect to the stellar distribution. This disk is regularly rotating out to $\sim 10 \mathrm{kpc}$ from the centre, but characterised by more irregular kinematics in the outer parts. The H I in NGC 3998 is likely the result of the merger with a relatively small galaxy $\left(M_{\text {star }} \sim 10^{8} M_{\odot}\right)$ which occurred about 1 Gyr ago and which has left no signature in the optical appearance of the galaxy.

3. The inner HI disk, together with the inner disk of ionised gas, is warping in a way mirroring the S-shape of the radio lobes and we suggest a connection between the two. We argue that the gas disk in the central regions has only recently (a few $\times 10^{6}$ yr ago) started to align with the stellar major axis. The effect of the stellar torques that case the warping of the disk could also cause the gas clouds in the central regions to lose angular momentum and fall into the super-massive black hole. We suggest that this mechanism may have triggered the radio continuum source, something we infer has happened on comparably short timescales. In this scenario, the $S$-shape of the radio structure would be due to the jet axis adapting to the changing angular momentum axis of the warping gas disk.

We predict additional large-scale, diffuse radio structures similar to those in NGC 3998 will be detected in other galaxies by new, deeper observations that are sensitive to low-surface-brightness emission. Future studies are planned to further explore the properties of the newly-discovered diffuse radio lobes of NGC 3998. These include observations with the Low Frequency Array (van Haarlem et al. 2013), the Giant Metrewave Radio Telescope, and the Karl G. Jansky Very Large Array that will allow a detailed analysis of the radio spectral indices of the continuum emission of the diffuse lobes of NGC 3998.

Acknowledgements. We thank Freeke van der Voort for useful discussions and suggestions during the preparation of this paper. We also thank J. R. SánchezGallego for providing the $\mathrm{H} \alpha$ image used in Fig. 6. The research leading to these results has received funding from the European Research Council under the European Union's Seventh Framework Programme (FP/2007-2013) / ERC Advanced Grant RADIOLIFE-320745. The Westerbork Synthesis Radio telescope is operated by the Netherlands Institute for Radio Astronomy (ASTRON) with support of the Netherlands Foundation for Scientific Research (NWO). We thank the referee for the constructive comments which have helped improve this paper.

\section{References}

Adelman-McCarthy, J. K., Agüeros, M. A., Allam, S. S., et al. 2008, ApJS, 175, 297

Alam, S., Albareti, F. D., Allende Prieto, C., et al. 2015, ApJS, 219, 12

Alatalo, K., Blitz, L., Young, L. M., et al. 2011, ApJ, 735, 88

Alatalo, K., Lacy, M., Lanz, L., et al. 2015, ApJ, 798, 31

Allen, S. W., Dunn, R. J. H., Fabian, A. C., Taylor, G. B., \& Reynolds, C. S. 2006, MNRAS, 372, 21

Argo, M. K., van Bemmel, I. M., Connolly, S. D., \& Beswick, R. J. 2015, MNRAS, 452, 1081

Baldi, R. D., \& Capetti, A. 2010, A\&A, 519, A48

Baldi, R. D., Capetti, A., \& Giovannini, G. 2015a, A\&A, 576, A38

Baldi, R. D., Giroletti, M., Capetti, A., et al. 2015b, A\&A, 574, A65

Balmaverde, B., Baldi, R. D., \& Capetti, A. 2008, A\&A, 486, 119

Best, P. N., \& Heckman, T. M. 2012, MNRAS, 421, 1569

Best, P. N., Kauffmann, G., Heckman, T. M., et al. 2005, MNRAS, 362, 25 Bondi, H. 1952, MNRAS, 112, 195

Boselli, A., Cortese, L., \& Boquien, M. 2014, A\&A, 564, A65

Brienza, M., Godfrey, L., Morganti, R., et al. 2016, A\&A, 585, A29

Briggs, D. S. 1995, Ph.D. Thesis, New Mexico Inst. Mining Tech.

Cappellari, M., Emsellem, E., Krajnović, D., et al. 2011a, MNRAS, 413, 813

Cappellari, M., Emsellem, E., Krajnović, D., et al. 2011b, MNRAS, 416, 1680

Cappellari, M., Scott, N., Alatalo, K., et al. 2013, MNRAS, 432, 1709 
Cavagnolo, K. W., McNamara, B. R., Nulsen, P. E. J., et al. 2010, ApJ, 720, 1066 Combes, F., García-Burillo, S., Casasola, V., et al. 2013, A\&A, 558, A124 Condon, J. J., Cotton, W. D., Greisen, E. W., et al. 1998, AJ, 115, 1693 Croston, J. H., Kraft, R. P., \& Hardcastle, M. J. 2007, ApJ, 660, 191

Croston, J. H., Hardcastle, M. J., Birkinshaw, M., Worrall, D. M., \& Laing, R. A. 2008, MNRAS, 386, 1709

Croston, J. H., Kraft, R. P., Hardcastle, M. J., et al. 2009, MNRAS, 395, 1999

Croton, D. J., Springel, V., White, S. D. M., et al. 2006, MNRAS, 365, 11 de Francesco, G., Capetti, A., \& Marconi, A. 2006, A\&A, 460, 439 den Heijer, M., Oosterloo, T. A., Serra, P., et al. 2015, A\&A, 581, A98 Devereux, N. 2011, ApJ, 727, 93

Duc, P.-A., Cuillandre, J.-C., Karabal, E., et al. 2015, MNRAS, 446, 120 Ekers, R. D., Fanti, R., Lari, C., \& Parma, P. 1978, Nature, 276, 588 Emonts, B. H. C., Morganti, R., Tadhunter, C. N., et al. 2006, A\&A, 454, 125 Eracleous, M., Hwang, J. A., \& Flohic, H. M. L. G. 2010, ApJ, 711, 796

Filho, M. E., Barthel, P. D., \& Ho, L. C. 2002, A\&A, 385, 425

Ford, H. C., Dahari, O., Jacoby, G. H., Crane, P. C., \& Ciardullo, R. 1986, ApJ, 311, L7

Gaspari, M., Brighenti, F., \& Temi, P. 2015, A\&A, 579, A62

González Delgado, R. M., Cid Fernandes, R., Pérez, E., et al. 2004, ApJ, 605, 127

González-Martín, O., Masegosa, J., Márquez, I., Guainazzi, M., \& Jiménez-Bailón, E. 2009, A\&A, 506, 1107

Gregory, P. C., Scott, W. K., Douglas, K., \& Condon, J. J. 1996, ApJS, 103, 427

Hardcastle, M. J., Evans, D. A., \& Croston, J. H. 2007, MNRAS, 376, 1849

Haynes, M. P., Giovanelli, R., \& Chincarini, G. L. 1984, ARA\&A, 22, 445

Heckman, T. M. 1980, A\&A, 87, 152

Helmboldt, J. F., Taylor, G. B., Tremblay, S., et al. 2007, ApJ, 658, 203

Hernández-García, L., González-Martín, O., Márquez, I., \& Masegosa, J. 2013, A\&A, 556, A47

Hibbard, J. E., van Gorkom, J. H., Rupen, M. P., \& Schiminovich, D. 2001, Gas and Galaxy Evolution, eds. J. E. Hibbard, M. Rupen, \& J. H. van Gorkom ASP Conf. Ser., 240

Ho, L. C., Filippenko, A. V., \& Sargent, W. L. W. 1997, ApJS, 112, 315

Hopkins, P. F., \& Quataert, E. 2011, MNRAS, 415, 1027

Hummel, E. 1980, A\&AS, 41, 151

Hummel, E., van der Hulst, J. M., \& Dickey, J. M. 1984, A\&A, 134, 207

Kharb, P., O'Dea, C. P., Baum, S. A., Colbert, E. J. M., \& Xu, C. 2006, ApJ, 652, 177

Kharb, P., O’Dea, C. P., Tilak, A., et al. 2012, ApJ, 754, 1

Knapp, G. R., van Driel, W., \& van Woerden, H. 1985, A\&A, 142, 1

Krajnović, D., Emsellem, E., Cappellari, M., et al. 2011, MNRAS, 414, 2923

Laing, R. A., \& Bridle, A. H. 2014, MNRAS, 437, 3405

Laing, R. A., Parma, P., de Ruiter, H. R., \& Fanti, R. 1999, MNRAS, 306, 513

Laing, R. A., Guidetti, D., Bridle, A. H., Parma, P., \& Bondi, M. 2011, MNRAS, 417, 2789

Maccagni, F. M., Morganti, R., Oosterloo, T. A., \& Mahony E. K. 2014, A\&A, 571, A67

Maccagni, F. M., Santoro, F., Morganti, R., et al. 2016, A\&A, 588, A46

Maddox, N., Hess, K. M., Obreschkow, D., Jarvis, M. J., \& Blyth, S.-L. 2015 MNRAS, 447, 1610

Maoz, D., Nagar, N. M., Falcke, H., \& Wilson, A. S. 2005, ApJ, 625, 699

Martini, P., Dicken, D., \& Storchi-Bergmann, T. 2013, ApJ, 766, 121

Martí-Vidal, I., Marcaide, J. M., Alberdi, A., et al. 2011, A\&A, 533, A111

Mason, R. E., Rodríguez-Ardila, A., Martins, L., et al. 2015, ApJS, 217, 13

Mathews, W. G., \& Brighenti, F. 2003, ARA\&A, 41, 191

McDermid, R. M., Alatalo, K., Blitz, L., et al. 2015, MNRAS, 448, 3484
Mezcua, M., Prieto, M. A., Fernández-Ontiveros, J. A., et al. 2015, MNRAS, 452, 4128

Morganti, R., Fanti, C., Fanti, R., Parma, P., \& de Ruiter, H. R. 1987, A\&A, 183, 203

Morganti, R., Oosterloo, T. A., Reynolds, J. E., Tadhunter, C. N., \& Migenes, V. 1997, MNRAS, 284, 541

Morganti, R., Holt, J., Tadhunter, C., et al. 2011, A\&A, 535, A97

Morganti, R., Oosterloo, T., Oonk, J. B. R., Frieswijk, W., \& Tadhunter, C. 2015, A\&A, 580, A1

Mueller Sánchez, F., Davies, R. I., Eisenhauer, F., et al. 2006, A\&A, 454, 481

Mueller-Sánchez, F., Prieto, M., Mezcua, M., et al. 2013, ApJ, 763, L1

Nagar, N. M., Falcke, H., \& Wilson, A. S. 2005, A\&A, 435, 521

Negri, A., Pellegrini, S., \& Ciotti, L. 2015, MNRAS, 451, 1212

Nemmen, R. S., \& Tchekhovskoy, A. 2015, MNRAS, 449, 316

Neumayer, N., Cappellari, M., Reunanen, J., et al. 2007, ApJ, 671, 1329

Nyland, K., Alatalo, K., Wrobel, J. M., et al. 2013, ApJ, 779, 173

Nyland, K., Young, L. M., Wrobel, J. M., et al. 2016, MNRAS, 458, 2221

Oosterloo, T., Verheijen, M. A. W., van Cappellen, W., et al. 2009, in Wide Field Astronomy \& Technology for the Square Kilometre Array, 70

O'Sullivan, S. P., Feain, I. J., McClure-Griffiths, N. M., et al., 2013, ApJ, 764, 162

Pak, M., Rey, S.-C., Lisker, T., et al. 2014, MNRAS, 445, 630

Perucho, M., Martí, J. M., Laing, R. A., \& Hardee,, P. E. 2014, MNRAS, 441, 1488

Pogge, R. W., Maoz, D., Ho, L. C., \& Eracleous, M. 2000, ApJ, 532, 323

Ptak, A., Terashima, Y., Ho, L. C., \& Quataert, E. 2004, ApJ, 606, 173

Russell, H. R., McNamara, B. R., Edge, A. C., et al. 2013, MNRAS, 432, 530

Sadler, E. M., Jenkins, C. R., \& Kotanyi, C. G. 1989, MNRAS, 240, 59

Sadler, E. M., Cannon, R. D., Mauch, T., et al. 2007, MNRAS, 381, 211

Sánchez-Gallego, J. R., Knapen, J. H., Wilson, C. D., et al. 2012, MNRAS, 422, 3208

Sanders, R. H., \& Verheijen, M. A. W. 1998, ApJ, 503, 97

Saripalli, L., Subrahmanyan, R., Thorat, K., et al. 2012, ApJS, 199, 27

Sault, R. J., Teuben, P. J., \& Wright, M. C. H. 1995, Astronomical Data Analysis Software and Systems IV, 77, 433

Serra, P., Oosterloo, T., Morganti, R., et al. 2012, MNRAS, 422, 1835

Shulevski, A., Morganti, R., Oosterloo, T., \& Struve, C. 2012, A\&A, 545, A91

Steenbrugge, K. C., \& Blundell, K. M. 2007, Ap\&SS, 310, 321

Struve, C., Oosterloo, T. A., Morganti, R., \& Saripalli, L. 2010a, A\&A, 515, A67

Struve, C., Oosterloo, T., Sancisi, R., Morganti, R., \& Emonts, B. H. C. 2010b, A\&A, 523, A75

Terashima, Y., Ho, L. C., \& Ptak, A. F. 2000, ApJ, 539, 161

Tully, R. B., Verheijen, M. A. W., Pierce, M. J., Huang, J.-S., \& Wainscoat, R. J. 1996, AJ, 112, 2471

van Cappellen, W. A., Bakker, L., \& Oosterloo, T. A. 2009, in 2009 IEEE Antennas and Propagation Society International Symposium, 1

van de Voort, F., Davis, T. A., Kereš, D., et al. 2015, MNRAS, 451, 3269

van Haarlem, M. P., Wise, M. W., Gunst, A. W., et al. 2013, A\&A, 556, A2

Walsh, J. L., van den Bosch, R. C. E., Barth, A. J., \& Sarzi, M. 2012, ApJ, 753, 79

Wild, V., Heckman, T., \& Charlot, S. 2010, MNRAS, 405, 933

Wrobel, J. M. 1991, AJ, 101, 127

Wrobel, J. M., \& Heeschen, D. S. 1984, ApJ, 287, 41

Wrobel, J. M., \& Heeschen, D. S. 1991, AJ, 101, 148

Younes, G., Porquet, D., Sabra, B., Reeves, J. N., \& Grosso, N. 2012, A\&A, 539, A104

Young, L. M., Bureau, M., Davis, T. A., et al. 2011, MNRAS, 414, 940

Yun, M. S., Ho, P. T. P., \& Lo, K. Y. 1994, Nature, 372, 530 